\title{
ANÁLISE DA SECAGEM DO BAGAÇO DE LARANJA EM CAMADA FINA UTILIZANDO MODELOS SEMI-TEÓRICOS E EMPÍRICOS
}

\author{
Leila Denise Fiorentin ${ }^{1}$ \\ Bruna Tozetto Menon ${ }^{2}$ \\ João Adriano Alves ${ }^{2}$ \\ Sueli Tereza Davantel de Barros ${ }^{2}$ \\ Nehemias Curvelo Pereira ${ }^{2}$ \\ Oswaldo Curtty da Motta Lima \\ Aparecido Nivaldo Modenes ${ }^{l}$
}

\begin{abstract}
Resumo: As características do processo de secagem do bagaço de laranja foram estudadas para a faixa de temperatura de 33 até $92^{\circ} \mathrm{C}$ e velocidade do ar constante. A dependência da difusividade com diferentes temperaturas foi descrita por uma equação do tipo Arrhenius onde se obteve um valor de energia de ativação de 10,699 $\mathrm{KJ} \mathrm{mol}^{-1}$. O processo de secagem também foi estudado por meio da aplicação de modelos semi empirícos descritos na literatura. Os valores estimados das constantes dos modelos para as diferentes temperaturas mostraram forte dependência com a temperatura inicial. $\mathrm{O}$ modelo de Page Modificado apresentou melhores resultados segundo os critérios estatísticos Teste $\mathrm{F}$ e $\mathrm{R}^{2}$, portanto, foi considerado o modelo que melhor se ajustou aos dados experimentais para todas as condiçôes de secagem estudadas. Assim, o modelo de Page Modificado pode ser utilizado para tarefas de otimização no processo de secagem de bagaço de laranja.
\end{abstract}

Palavras-chaves: bagaço de laranja; secagem; modelagem; camada fina.

Abstract: Important physical characteristics of orange bagasse for biosorption processes were evaluated within a 33-92 ${ }^{\circ} \mathrm{C}$ drying temperature range under controlled air velocity, considering some semi-empirical models to represent the experimental data. It was noticed that the particle diffusivity into the biomass is a strong depending parameter on the drying temperature. By the application of an Arrhenius-type equation on the diffusivity data, a value of $10.699 \mathrm{KJ} \mathrm{mol}^{-1}$ for the activation energy was attained. In addition, the drying process data were interpreted by using unstructural models, showing the strongly drying temperature depending behavior for all model constant parameters. Regarding the whole of modeling results, the modified Page model has fitted well to the drying process data, according to some statistical parameters such as F-test, which were better than the other ones. Based on such results, the modified Page model was thus assigned the best one to represent the experimental data as well as it could be recommended as an adequate guide model for the drying process optimization tests of orange bagasse among other similar biomasses.

Keywords: orange bagasse; drying; modeling; thin layer.

\footnotetext{
${ }^{1}$ Departamento de Engenharia Química, Universidade Estadual do Oeste do Paraná.

${ }^{2}$ Departamento de Engenharia Química, Universidade Estadual de Maringá.
} 


\section{INTRODUÇÃO}

A laranja (Citrus sinensis L. Osbeck - família Rutaceae) é uma fruta muito conhecida mundialmente por apresentar quantidades consideráveis de sais minerais e vitaminas, sendo seu principal produto o suco de laranja.

Entretanto, há outros subprodutos que podem ser explorados por apresentarem um elevado valor comercial. Um dos subprodutos da indústria de extração de suco de laranja com o menor valor agregado é o resíduo denominado industrialmente de bagaço da laranja úmido, que corresponde a 42\%, (ÍTAVO et al., 2000) do total da fruta, sendo composto pela casca, filamentos de membranas, albedo e sementes. Este resíduo pode causar diversos problemas ambientais e econômicos, devido à elevada fermentação e degradabilidade, resultante de sua alta umidade, acima de $85 \%$ (b.u.), (TRIPODO et al., 2004). O bagaço é rico em açúcares, fibras, carboidratos solúveis e pectina, e, desidratado, é empregado na produção de ração animal. Entretanto, o processo de secagem geralmente apresenta um elevado custo tornando-se muitas vezes inviável economicamente (ÍTAVO et. al., 2000). Deste modo, há interesse no desenvolvimento de outras aplicaçôes para o bagaço da laranja, ampliando assim o mercado deste resíduo.

Uma possível aplicação é no processo de adsorção, pois, conforme ARAMI et al. (2005) e SIVARAJ et al. (2001), a casca da laranja apresenta característica adsortiva semelhante a do carvão ativado para a remoção de corantes. FIORENTIN et al. (2010) verificaram que o bagaço da laranja, remove satisfatoriamente determinados tipos de corantes presentes nos efluentes de indústrias têxteis.

A utilização de biosorventes oriundos de resíduos agrícolas com elevado teor de umidade requer a remoção parcial ou total da água presente nos poros do sólido. O procedimento de secagem assegura a qualidade e estabilidade do produto, diminuindo a atividade biologia e as mudanças químicas e físicas que podem vir a ocorrer durante o armazenamento do material. A secagem de um material sólido envolve simultaneamente a transferência de calor e de massa (MWITHIGA e OLWAL, 2005, GHAZANFARI, et al., 2006, ALMEIDA et al., (1999), SOUSA, 2006, CORRÊA, et al., 2006) e seu com- portamento é caracterizado pela perda do teor de umidade em função do tempo. $O$ processo de secagem pode ser acelerado com a passagem de ar forçado pela amostra, denominado de secagem convectiva forçada.

Devido a forma, tamanho e estrutura interna, os resíduos agrícolas são muito diferentes entre si e quando o produto é colocado em contato com o ar de secagem, ocorre a contração de volume do material. Desta forma, essa variação no volume de poros pode vir a interferir no processo de adsorção. A movimentação da umidade no interior dos materiais vai depender do tipo do resíduo que está sendo submetido à secagem. Em produtos capilares porosos, como a maioria dos produtos agrícolas, pode-se destacar os possíveis mecanismos de transporte como sendo o de difusão liquida, difusão capilar na superfície, fluxo hidrodinâmico, difusão de vapor e difusão térmica (MARTINAZZO, 2007).

A cinética de secagem de vários produtos pode ser descrita por modelos matemáticos semi-empíricos ou puramente empíricos (MADAMBA, 2003). Os modelos semi-empíricos se baseiam na analogia com a Lei de Newton para o resfriamento, aplicada à transferência de massa, enquanto que os modelos empíricos apresentam uma boa relação entre o conteúdo médio do teor de umidade e o tempo de secagem e consideram como mecanismo principal a difusão baseada na Segunda Lei de Fick (CRANK, 1975 citado em YILDIRIM et. al., 2011). O coeficiente de difusão e a energia de ativação são dois parâmetros importantes a serem calculados com base na segunda lei de Fick. O coeficiente de difusividade efetiva pode ser calculado utilizando um modelo simplificado que relaciona um termo exponencial do tipo Arrhenius que apresenta dependência com o teor de umidade e a temperatura (RIVERA et al., 2007).

Desta forma, este trabalho tem por objetivos: analisar o comportamento da secagem convectiva do bagaço de laranja em cinco diferentes temperaturas, mantendo a velocidade do ar constante; aplicar modelos matemáticos da literatura aos dados experimentais; selecionar o modelo que melhor representa o comportamento dos dados por meio de métodos estatísticos e determinar o coeficiente de difusividade e a energia de ativação para o resíduo. 


\section{MATERIAIS E MÉTODOS}

\subsection{MATERIAIS}

O bagaço de laranja foi cedido por uma indústria processadora de suco de laranja da região de Maringá, Paraná - Brasil. O resíduo industrial, livre do suco e óleos essenciais, foi coletado e armazenado em sacos plásticos em um congelador à temperatura de $-15^{\circ} \mathrm{C}$ no laboratório. Para sua utilização, o bagaço foi descongelado naturalmente e triturado em liquidificador.

\subsection{EXPERIMENTOS DE SECAGEM}

O módulo experimental utilizado para a secagem do bagaço da laranja consiste de um secador de laboratório com ajuste de velocidade, ligado a um duto metálico, equipado com quatro resistências elétricas. À saída do duto metálico é conectada a uma caixa de aço por onde passa o ar aquecido através de uma tela metálica, conforme mostrado na Figura 1. A temperatura de secagem da amostra é ajustada a partir de resistências elétricas, controladas por um reostato. $\mathrm{O}$ indicador de temperatura consiste de um termopar inserido logo abaixo da tela perfurada do secador.

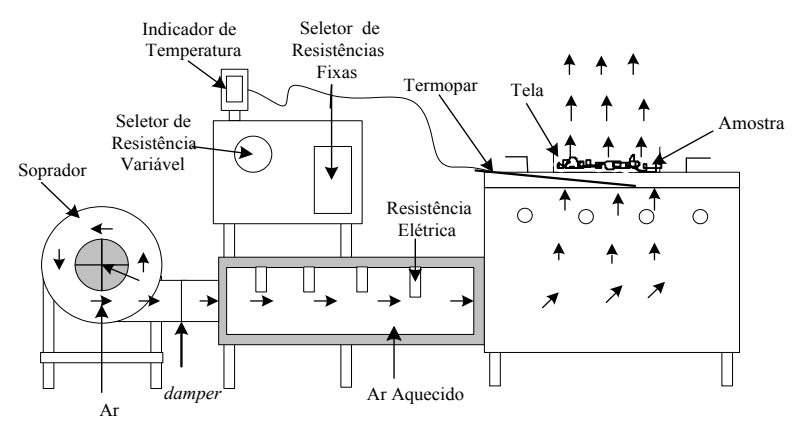

Figura 1. Módulo experimental, sistema convectivo (LUZ et. al., 2006)

Após o descongelamento e trituração do resíduo, $150 \mathrm{~g}$ do material foi colocado sobre a tela metálica formando uma camada de aproximadamente $10 \mathrm{~mm}$ de espessura. Em seguida, ajustou-se a temperatura e velocidade do ar desejada e a amostra foi pesada em balança analítica $( \pm 0,0001 \mathrm{~g})$ em períodos de tempo pré-determinados até massa constante. Durante os experimentos foram realizadas medições da temperatura em vários pontos da superfície da amostra que se encontrava sobre a tela, garantindo assim a temperatura uniforme na amostra. $\mathrm{O}$ bagaço foi seco nas temperaturas de $33,41,50,66,80$ e $92{ }^{\circ} \mathrm{C}$ com velocidade do ar constante de $1,3 \mathrm{~m} \mathrm{~s}^{-1}$. Durante os experimentos (triplicata) determinou-se a velocidade do ar de secagem com um anemômetro digital e a umidade relativa ambiente com um psicrômetro digital.

O teor de umidade em base seca, calculado pela Equação 1 foi determinado com o auxílio do método da estufa, que consiste em colocar uma determinada massa de amostra em estufa na temperatura de $105^{\circ} \mathrm{C}$, por 24 horas.

$X(b . s)=\frac{M_{\text {úmida }}-M_{\mathrm{sec} a}}{M_{\mathrm{sec} a}}$

em que $\mathrm{X}$ (b.s) é o teor de umidade (água) da amostra em base seca, ( $\mathrm{g} \mathrm{H}_{2} \mathrm{O} / \mathrm{g}$ sól. seco), $\mathrm{M}_{\text {úmida- }}$ $-\mathrm{M}_{\mathrm{seca}}$ é a massa de água presente na amostra, $(\mathrm{g})$ e

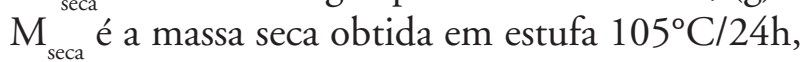
(g).

\subsection{MODELAGEM DO PROCESSO DE SECAGEM}

Segundo MADAMBA et al. (2003), pode-se utilizar um modelo análogo à lei do resfriamento de Newton, que considera apenas a resistência superficial no sólido e assume que a taxa de secagem é proporcional à diferença entre o teor de umidade em um determinado tempo e o teor de umidade de equilíbrio, (GHAZANFARI, et al., 2006) como mostra a Equação 2.

$-\frac{d X}{d t}=k\left(X-X_{e q}\right)$

em que $\mathrm{X}$ é o teor de umidade do produto em um determinado tempo, ( $\mathrm{g} \mathrm{H}_{2} \mathrm{O} / \mathrm{g}$ sól. seco min), $\mathrm{X}_{\mathrm{eq}}$ é o teor de umidade de equilíbrio determinado experimentalmente ( $\mathrm{g} \mathrm{H}_{2} \mathrm{O} / \mathrm{g}$ sól. seco) e k é a constante de secagem, $\left(\mathrm{min}^{-1}\right)$.

O teor de umidade de equilíbrio foi determinado por meio do teor de umidade inicial do bagaço, calculando-se a massa seca da amostra baseada no teor da umidade inicial do bagaço, como mostra a Equação 3.

$M_{\mathrm{sec} a}=M_{t}-\frac{X(b . s) M_{t}}{100}$

em que, $M_{t}$ é a massa total de amostra úmida utilizada em cada condição $\left(\mathrm{g} \mathrm{H}_{2} \mathrm{O}\right)$. 
Para a determinação do teor de umidade de equilíbrio $\left(\mathrm{X}_{\mathrm{eq}}\right)$ utilizou-se a razão entre a massa úmida $\left(\mathrm{M}_{\text {úmida }}\right)$ e a massa seca $\left(\mathrm{M}_{\text {seca }}\right)$. A $\mathrm{X}_{\text {eq }}$ considerada para o cálculo do teor de umidade do produto $\left(\mathrm{X}_{\mathrm{R}}\right)$ era quando a razão $\mathrm{M}_{\text {úmida }} / \mathrm{M}_{\text {seca }}$ tornava-se constante. Desta forma, para cada condição de secagem a amostra possuía um teor de umidade de equilíbrio equivalente.

A curva de secagem apresenta basicamente dois períodos, um de taxa constante, no qual a superfície do material está com um nível elevado de umidade e outro de taxa decrescente de secagem, que pode ser divido em duas fases distintas, a depender do comportamento da taxa de secagem. Segundo SOUSA (2003), no primeiro período é sugerido como principal mecanismo de transporte, o escoamento capilar, a difusão de líquido e a difusão de vapor, enquanto que, no segundo período, a evaporação da umidade ocorre no interior do material e a difusão do vapor formado pode dominar os mecanismos de transporte de líquido. Assim, pode-se utilizar o modelo da difusão com a Segunda Lei de Fick (Crank, 1975), para descrever os dados de secagem e determinar o coeficiente de difusividade, conforme a Equação 4. Quando se aplica esta lei, considera-se que as condições são isotérmicas e que a resistência à transferência de úmida ocorre somente na superfície do produto (BROOKER et al., 1992)

$$
\frac{\partial X}{\partial t}=D_{e f f}\left(\nabla^{2} X\right)
$$

em que $\mathrm{D}_{\text {eff }}$ é o coeficiente de difusividade efetiva $\left(\mathrm{m}^{2} \mathrm{~s}^{-1}\right)$.

Assumindo, na Equação 4, que a migração da umidade do interior do material para a superfície ocorre somente por difusão, mantendo constantes a temperatura e o coeficiente de difusividade e desprezando o encolhimento do material, a solução tem a forma da Equação 5, (DOYMAZ, 2007).

$$
\begin{array}{r}
X_{R}=\frac{X-X_{e q}}{X_{0}-X_{e q}}=\frac{8}{\pi^{2}} \sum_{n=0}^{n=\infty} \frac{1}{(2 n+1)^{2}} \\
\exp \left[-\frac{(2 n+1)^{2} \pi^{2} D_{e f f} t}{4 L^{2}}\right]
\end{array}
$$

em que $\mathrm{D}$ é o coeficiente de difusividade efetiva $\left(\mathrm{m}^{2} \mathrm{~s}^{-1}\right)$ e L é a metade da espessura da amostra (m) e t é o tempo de secagem (s).
A partir da solução geral da Segunda Lei de Fick, Equação 5, casos particulares que envolvem a dependência da constante de secagem com o tempo em determinadas condições iniciais, podem ser obtidos, tais como os modelos semi-empíricos de Dois Termos (1974), Lewis (1921), Page (1949) e Wang e Singh (1978), os quais foram aplicados na modelagem das curvas de secagem obtidas para o bagaço de laranja (HENDERSON, 1974, LEWIS, 1921, PAGE, 1949, WANG e SINGH, 1978).

ORIKASA, et al. (2008) e GHAZANFARI et al. (2006) mostraram que com a integração da Equação 2 e assumindo as condições iniciais $t=0$, $\mathrm{X}=\mathrm{X}_{0}$, tem-se a Equação 6, conhecida como modelo de Lewis (1921). Este modelo geralmente é utilizado para prever o comportamento das curvas de secagem de produtos agrícolas com elevada quantidade de açúcar, em camada fina.

$$
X_{R}=\frac{X-X_{e q}}{X_{0}-X_{e q}}=e^{-k t}
$$

em que $\mathrm{X}_{\mathrm{R}}$ é o teor de umidade do produto no tempo t ( $\mathrm{g} \mathrm{H}_{2} \mathrm{O} / \mathrm{g}$ sol. seco min) $\mathrm{X}_{0}$ é o teor de umidade inicial, $\left(\mathrm{g} \mathrm{H}_{2} \mathrm{O} / \mathrm{g}\right.$ sol. seco), $\mathrm{k}$ é a constante de secagem $\left(\mathrm{min}^{-1}\right)$ e t é o tempo de secagem, (min).

O modelo da difusão de Dois Termos (1974) mostra os primeiros dois termos da solução em série da Segunda Lei de Fick, Equação 4. Segundo DANDAMRONGRAK et al. (2002), este modelo foi criado na tentativa de melhorar a precisão dos modelos já existentes, especialmente no período final de secagem. Este modelo pode ser aplicado a qualquer tipo de geometria de partícula e condições de contorno, considerando-se que a difusividade é constante. O modelo de Dois Termos (1974), Equação 7, é recomendado para prever o comportamento dos dados de secagem de produtos agrícolas (DOYMAZ, 2006).

$$
X_{R}=A \exp (-k t)+B \exp \left(-k_{0} t\right)
$$

em que A, B, são os parâmetros dos modelos, $\mathrm{k}, \mathrm{k}_{0}$ são as constantes de secagem $\left(\mathrm{min}^{-1}\right)$.

Segundo OLIVEIRA et. al. (2006), em algumas situações a teoria difusional não é adequada para ajustar o comportamento dos dados ex- 
perimentais, quando há uma elevada interferência da resistência interna do material no processo de secagem. Assim, o modelo de Page (1949) (Page, 1949), Equação 8, pode ser aplicado para tais situações.

$$
X_{R}=\exp \left(-k t^{n}\right)
$$

em que n é um parâmetro do modelo.

O modelo de Wang e Singh (1978), Equação 9, foi inicialmente aplicado no estudo da secagem de casca de arroz. Assim como os demais modelos empíricos de secagem este modelo apresenta uma relação direta entre o teor de água e o tempo de secagem, omitindo características fundamentais que acontecem durante o período de secagem, entretanto consegue se ajustar às curvas de secagem em determinadas condiçôes experimentais. Este modelo é muito usado em estudos de secagem de produtos agrícolas e de alimentos (MARTINAZZO et al., 2007).

$$
X_{R}=A t^{2}+B t+1
$$

em que $\mathrm{A}, \mathrm{B}$ são parâmetros do modelo

Os modelos que melhor se ajustaram aos dados experimentais foram escolhidos com base nos maiores valores do coeficiente de determinação $\left(R^{2}\right)$ e do Teste $F$, e nos menores valores da raiz do erro médio ao quadrado (RMSE), (SOUSA, 2003, ERTEKIN e YALDIZ, 2004, DOYMAZ, 2007), como mostram as Equações 10 e 11.

$R M S E=\left[\frac{1}{N} \sum_{i=1}^{N}\left(X_{\mathrm{exp}, i}-X_{p r e d, i}\right)^{2}\right]^{1 / 2}$

Teste $F=\frac{\sum \bar{X}_{\text {pred }, i}^{2}}{\sum \bar{X}_{r e s i d, i}^{2}}$

em que $\mathrm{X}_{\text {exp,i }}$ é o teor de umidade obtida experimentalmente $\left(\mathrm{g} \mathrm{H}_{2} \mathrm{O} / \mathrm{g}\right.$ sól. seco), $\mathrm{X}_{\text {pred,i }}$ é o teor de umidade predita pelo modelo $\left(\mathrm{g} \mathrm{H}_{2}^{\text {pred, }} \mathrm{O} / \mathrm{g}\right.$ sól. seco), $\mathrm{X}_{\text {resd,i }}$ é o teor de umidade residual $\left(\mathrm{g} \mathrm{H}_{2} \mathrm{O} / \mathrm{g}\right.$ sól. seco) definida como a diferença entre o $\mathrm{X}_{\text {exp,i }}$ e o $X_{\text {pred,i }}$ e $\mathrm{N}$ o número de experimentos.

\subsection{DETERMINAÇÃO DO COEFICIENTE DE DIFUSIVIDADE E DA ENERGIA DE ATIVAÇÃO}

Para tempos longos de secagem $\left(\frac{D_{e f f} t}{4 L^{2}}>2\right) \begin{aligned} & \text {, a solução geral da Segunda Lei de } \\ & \text { Fick, Equação } 5 \text { pode ser reduzida a } \\ & \text { apenas o primeiro termo da série }\end{aligned}$ (DOYMAZ, 2007), obtendo-se a Equação 12.

$$
X_{R}=\frac{8}{\pi^{2}} \exp \left(-\frac{\pi^{2} D_{e f f} t}{4 L^{2}}\right)
$$

Linearizando a Equação 12, obtém-se a inclinação $\left(\mathrm{K}_{0}\right)$ de acordo com a Equação 13.

$$
K_{0}=\frac{\pi^{2} D_{e f f}}{4 L^{2}}
$$

Segundo SOUSA, (2003) o estudo dos processos de secagem baseado na utilização do modelo da difusão Fick estaria fundamentado na análise dos efeitos globais do fenômeno no interior do material, não apresentando condições para uma descrição dos possíveis mecanismos internos de migração de água. O coeficiente global de transferência, denominado difusividade efetiva, englobaria todos os possíveis efeitos internos, sendo dependente do teor de umidade e da temperatura.

$D_{\text {eff }}=D_{0} \exp \left(-\frac{E_{a c t}}{R T}\right)$

em que $\mathrm{D}_{0}$ é o fator pré-exponencial da equação de Arrhenius $\left(\mathrm{m}^{2} \mathrm{~s}^{-1}\right)$, $\mathrm{R}$ é a constante universal dos gases $\left(8,314 \mathrm{~kJ} \mathrm{~mol}^{-1} \mathrm{~K}^{-1}\right)$, $\mathrm{T}$ a temperatura absoluta $(\mathrm{K})$ e $\mathrm{E}_{\text {act }}$ a energia de ativação $\left(\mathrm{kJ} \mathrm{mol}^{-1}\right)$.

\section{RESULTADOS E DISCUSSŌES}

\subsection{MODELAGEM DAS CURVAS DE SECAGEM}

O teor de umidade de equilíbrio das amostras secas foi calculado levando-se em consideração a massa úmida da amostra e variou na faixa entre 0,02 e 0,12 g água/ $g$ sol. em base seca.

Com a realização da secagem convectiva do bagaço de laranja nas temperaturas de 33 a $92^{\circ} \mathrm{C}$, 
foi possível obter as curvas de secagem experimental e aplicar aos dados os modelos empíricos encontrados na literatura, como mostram as Figuras 2 a 7. Os parâmetros dos modelos foram obtidos por regressão não linear (Quasi-Newton) com o auxílio do software Statistica $6.0^{\circledR}$.

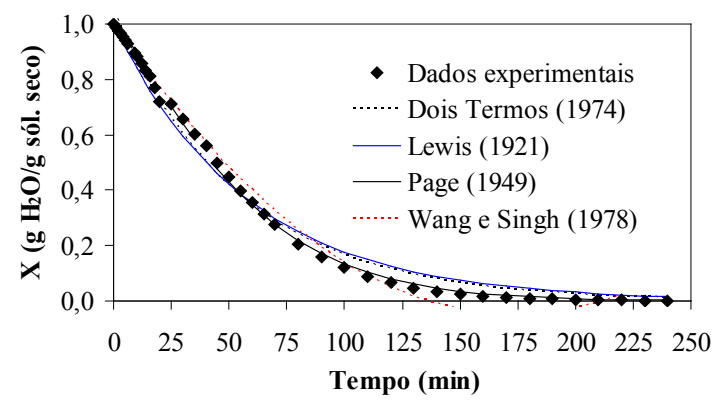

Figura 2. Ajuste de modelos matemáticos para a temperatura de $33 \pm 1^{\circ} \mathrm{C}$

As curvas de secagem obtidas experimentalmente mostram um comportamento típico, em que, o aumento de temperatura causa uma diminuição no tempo de secagem. Na Figura 2, a secagem do bagaço a temperatura de $33 \pm 1^{\circ} \mathrm{C}$ demorou cerca de $240 \mathrm{~min}$ para atingir o teor de umidade de equilíbrio, enquanto que, na Figura 7 para a máxima temperatura experimental de secagem, $92 \pm 1^{\circ} \mathrm{C}$, o tempo de secagem foi de aproximadamente $80 \mathrm{~min}$. Os resultados obtidos estão de acordo com MWITHIGA et al. (2005), MARTINAZZO et al. (2007), ORIKASA et al. (2008), DOYMAZ, (2007), KINGSLY e SIN$\mathrm{GH}$, (2007), que avaliaram o comportamento da secagem de alimentos em função do tempo e obtiveram resultados semelhantes.

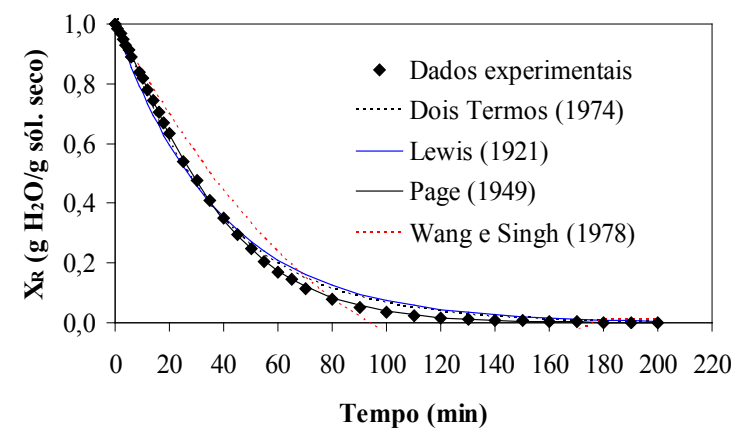

Figura 3. Ajuste de modelos matemáticos para a temperatura de $41 \pm 1^{\circ} \mathrm{C}$

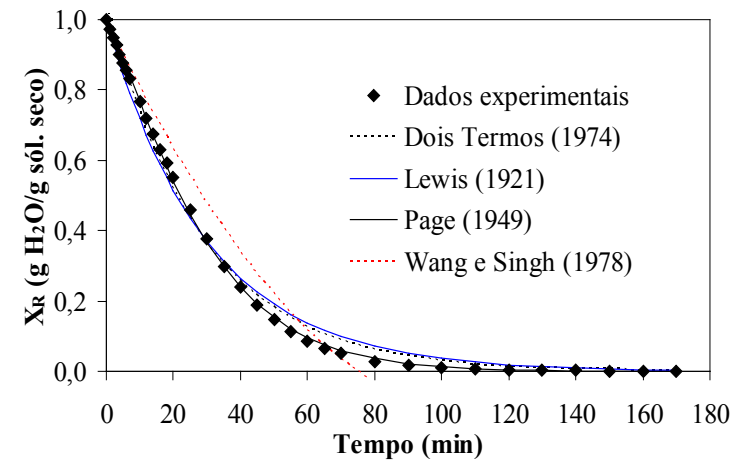

Figura 4. Ajuste de modelos matemáticos para a temperatura de $50 \pm 1^{\circ} \mathrm{C}$

Pela análise dos dados experimentais verifica-se, que o processo de secagem do bagaço da laranja apresenta dois períodos distintos de secagem baseados no comportamento da velocidade de secagem. Inicialmente se tem um período de acondicionamento do material ao fluxo de ar convectivo, em seguida um período de taxa constante com um período posterior de taxa decrescente, onde se tem o material seco naquelas condições. Este comportamento mostra que o ajuste dos resultados experimentais pode ser realizado pela Segunda Lei da difusão de Fick (KHRAISHEH, et al., 1997).

Em relação aos modelos matemáticos, observa-se nas Figuras 2 a 7 que o modelo de Wang e Singh (1978), com exceção dos dados experimentais da temperatura de $33^{\circ} \mathrm{C} \pm 1^{\circ} \mathrm{C}$ não conseguiu prever satisfatoriamente os dados experimentais principalmente no período inicial de secagem, onde se remove a umidade superficial do sólido.

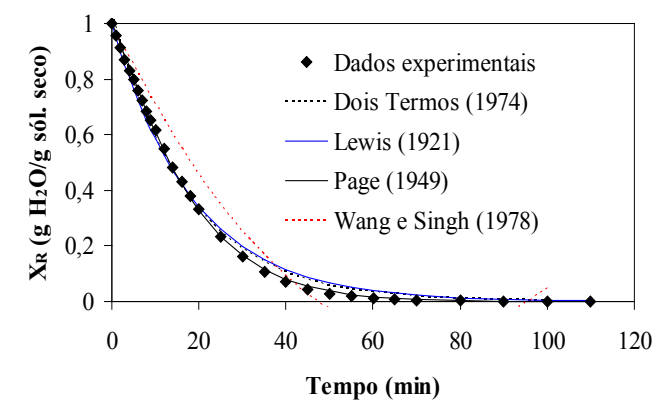

Figura 5. Ajuste de modelos matemáticos para a temperatura de $66 \pm 1^{\circ} \mathrm{C}$

Os modelos Dois termos (1974) e Lewis (1921) conseguiram uma melhor previsão em relação aos modelos de Wang e Singh (1978) para o período inicial do processo de secagem. Entretanto, observa-se que o modelo de Page (1949) é o que melhor representou os dados experimen- 
tais, em relação aos demais modelos em todas as temperaturas testadas, desde o período inicial de acondicionamento do material até o período final da secagem do bagaço de laranja.

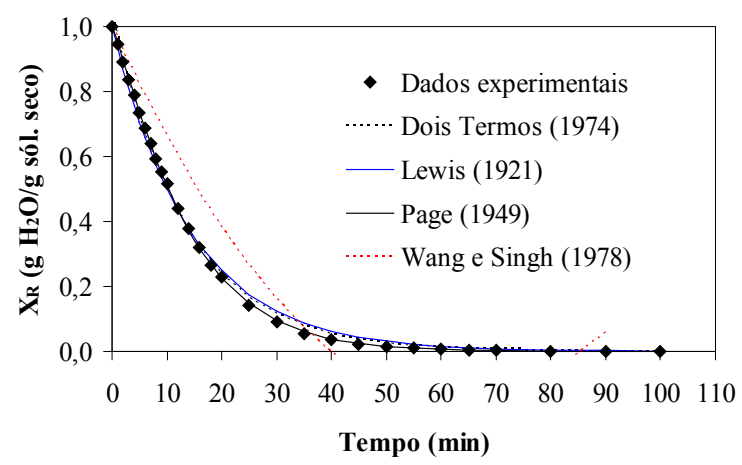

Figura 6. Ajuste de modelos matemáticos para a temperatura de $80 \pm 1{ }^{\circ} \mathrm{C}$

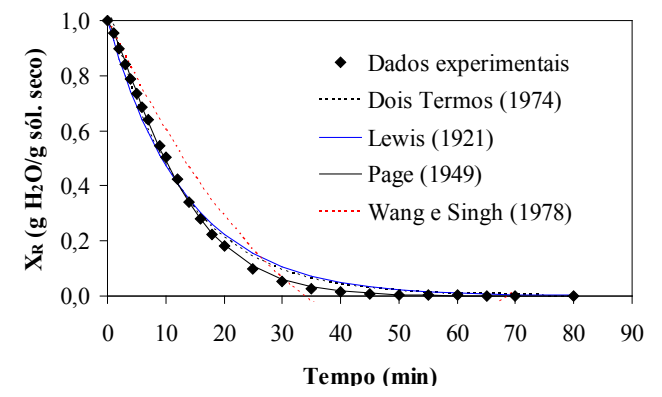

Figura 7. Ajuste de modelos matemáticos para a temperatura de $92 \pm 1^{\circ} \mathrm{C}$
$\mathrm{Na}$ Tabela 1 são mostrados os valores estatísticos do RMSE, Teste F e do coeficiente de determinação $\mathrm{R}^{2}$, que foram utilizados como critério de escolha do modelo que melhor representa os dados experimentais, bem como os valores dos teores de umidade inicial e final para cada modelo. Nota-se na Tabela 1 que o modelo de Page (1949) apresentou os maiores valores de $\mathrm{R}^{2}$ e do Teste $\mathrm{F}$ e os menores valores do RMSE, mostrando assim, o seu elevado potencial de representação dos dados experimentais em todas as temperaturas testadas. O modelo de Wang e Singh (1978) foi o que apresentou os mais baixos valores em relação ao Teste F, $\mathrm{R}^{2}$ e maiores valores de RMSE. Os modelos de Dois Termos (1974) e de Lewis (1921) apresentaram a mesma tendência em relação aos valores do Teste F, RMSE e R ${ }^{2}$. No entanto, o modelo de Dois Termos (1974) em todos os valores preditos do teor de umidade inicial $\left(\mathrm{X}_{0}\right)$, apresentou valores de umidade maior do que 1,0 (um), valores estes não aceitáveis.

Tabela 1. Comparação estatística do comportamento dos modelos

\begin{tabular}{|c|c|c|c|c|c|c|}
\hline $\mathbf{T}\left({ }^{\circ} \mathrm{C}\right)$ & Modelo & $\begin{array}{c}\mathrm{X}_{0} \\
\left(\mathrm{~g} \mathrm{H}_{2} \mathrm{O} / \mathrm{g}\right. \\
\text { sól. seco) }\end{array}$ & $\begin{array}{c}\mathrm{X}_{\mathrm{f}} \\
\left(\mathrm{g} \mathrm{H}_{2} \mathrm{O} / \mathrm{g}\right. \\
\text { sól. seco) }\end{array}$ & Teste F & RMSE & $\mathbf{R}^{2}$ \\
\hline 33 & Dois Termos (1974) & 1,047 & 0,013 & 311,239 & 0,0329 & 0,9924 \\
\hline 33 & Lewis (1921) & 1,000 & 0,016 & 206,395 & 0,0393 & 0,9892 \\
\hline 33 & Page (1949) & 1,000 & 0,000 & 1771,026 & 0,0137 & 0,9993 \\
\hline 33 & Wang e Singh (1978) & 1,000 & 0,050 & 267,717 & 0,0632 & 0,9908 \\
\hline $\mathbf{T}\left({ }^{\circ} \mathbf{C}\right)$ & Modelo & $\begin{array}{c}\mathrm{X}_{0} \\
\left(\mathrm{~g} \mathrm{H}_{2} \mathrm{O} / \mathrm{g}\right. \\
\text { sól. seco) }\end{array}$ & $\begin{array}{c}\mathrm{X}_{\mathrm{f}} \\
\left(\mathrm{g} \mathrm{H}_{2} \mathrm{O} / \mathrm{g}\right. \\
\text { sól. seco) }\end{array}$ & Teste F & RMSE & $\mathbf{R}^{2}$ \\
\hline 41 & Dois Termos (1974) & 1,049 & 0,004 & 564,482 & 0,0232 & 0,9981 \\
\hline 41 & Lewis (1921) & 1,000 & 0,005 & 296,242 & 0,03106 & 0,9931 \\
\hline 41 & Page (1949) & 1,000 & 0,000 & 21930,317 & 0,00373 & 0,9999 \\
\hline 41 & Wang e Singh (1978) & 1,000 & 0,070 & 72,471 & 0,06781 & 0,9670 \\
\hline
\end{tabular}




\begin{tabular}{|c|c|c|c|c|c|c|}
\hline $\mathbf{T}\left({ }^{\circ} \mathbf{C}\right)$ & Modelo & $\begin{array}{c}\mathrm{X}_{0} \\
\left(\mathrm{~g} \mathrm{H}_{2} \mathrm{O} / \mathrm{g}\right. \\
\text { sól. seco) }\end{array}$ & $\begin{array}{c}\mathrm{X}_{\mathrm{f}} \\
\left(\mathrm{g} \mathrm{H}_{2} \mathrm{O} / \mathrm{g}\right. \\
\text { sól. seco) }\end{array}$ & Teste F & RMSE & $\mathbf{R}^{2}$ \\
\hline 50 & Dois Termos (1974) & 1,045 & 0,003 & 422,931 & 0,0262 & 0,9949 \\
\hline 50 & Lewis (1921) & 1,000 & 0,003 & 266,007 & 0,0321 & 0,9925 \\
\hline 50 & Page (1949) & 1,000 & 0,000 & 4604,614 & 0,008 & 0,9997 \\
\hline 50 & Wang e Singh (1978) & 1,000 & 0,080 & 56,1550 & 0,0765 & 0,9574 \\
\hline $\mathbf{T}\left({ }^{\circ} \mathbf{C}\right)$ & Modelo & $\begin{array}{c}\mathrm{X}_{0} \\
\left(\mathrm{~g} \mathrm{H}_{2} \mathrm{O} / \mathrm{g}\right. \\
\text { sól. seco) }\end{array}$ & $\begin{array}{c}\mathrm{X}_{\mathrm{f}} \\
\left(\mathrm{g} \mathrm{H}_{2} \mathrm{O} / \mathrm{g}\right. \\
\text { sól. seco) }\end{array}$ & Teste F & RMSE & $\mathbf{R}^{2}$ \\
\hline 66 & Dois Termos (1974) & 1,041 & 0,003 & 567,261 & 0,0224 & 0,9960 \\
\hline 66 & Lewis (1921) & 1,000 & 0,002 & 365,125 & 0,0268 & 0,9942 \\
\hline 66 & Page (1949) & 1,000 & 0,000 & 4764,869 & 0,0108 & 0,9997 \\
\hline 66 & Wang e Singh (1978) & 1,000 & 0,050 & 40,238 & 0,0906 & 0,9340 \\
\hline $\mathrm{T}\left({ }^{\circ} \mathrm{C}\right)$ & Modelo & $\begin{array}{c}\mathrm{X}_{0} \\
\left(\mathrm{~g} \mathrm{H}_{2} \mathrm{O} / \mathrm{g}\right. \\
\text { sól. seco) }\end{array}$ & $\begin{array}{c}\mathrm{X}_{\mathrm{f}} \\
\left(\mathrm{g} \mathrm{H}_{2} \mathrm{O} / \mathrm{g}\right. \\
\text { sól. seco) }\end{array}$ & Teste F & RMSE & $\mathbf{R}^{2}$ \\
\hline 80 & Dois Termos (1974) & 1,037 & 0,000 & 1026,360 & 0,0153 & 0,9989 \\
\hline 80 & Lewis (1921) & 1,000 & 0,001 & 583,760 & 0,01986 & 0,9966 \\
\hline 80 & Page (1949) & 1,000 & 0,000 & 22524,984 & 0,00327 & 0,9999 \\
\hline 80 & Wang e Singh (1978) & 1,000 & 0,060 & 20,821 & 0,1230 & 0,8694 \\
\hline $\mathrm{T}\left({ }^{\circ} \mathrm{C}\right)$ & Modelo & $\begin{array}{c}\mathrm{X}_{0} \\
\left(\mathrm{~g} \mathrm{H}_{2} \mathrm{O} / \mathrm{g}\right. \\
\text { sól. seco) }\end{array}$ & $\begin{array}{c}\mathrm{X}_{\mathrm{f}} \\
\left(\mathrm{g} \mathrm{H} \mathrm{H}_{2} \mathrm{O} / \mathrm{g}\right. \\
\text { sól. seco) }\end{array}$ & Teste F & RMSE & $\mathbf{R}^{2}$ \\
\hline 92 & Dois Termos (1974) & 1,060 & 0,002 & 366,617 & 0,0260 & 0,9945 \\
\hline 92 & Lewis (1921) & 1,000 & 0,002 & 209,421 & 0,0334 & 0,9909 \\
\hline 92 & Page (1949) & 1,000 & 0,000 & 5497,880 & 0,00675 & 0,9998 \\
\hline 92 & Wang e Singh (1978) & 1,000 & 0,020 & 33,016 & 0,0946 & 0,9271 \\
\hline
\end{tabular}

$\mathrm{Na}$ Tabela 2 são mostrados os valores das constantes e dos coeficientes de secagem para o modelo de Page (1949), que segundo as Figuras 2 a 7 e a Tabela 1, foi o modelo que melhor representou os dados experimentais de secagem do bagaço da laranja. Observa-se que, a constante de secagem $\mathrm{k}$ aumenta com o aumento da temperatura. Nas condições testadas, os coeficientes de secagem para o modelo de Page (1949) apresentaram valor mínimo de $0,0062 \mathrm{~min}^{-1}$ na temperatura de $33 \pm 1^{\circ} \mathrm{C}$ e valor máximo de $0,0506 \mathrm{~min}^{-1}$ em $92 \pm 1^{\circ} \mathrm{C}$.
Tabela 2. Valores estimados dos parâmetros para o modelo de Page (1949)

\begin{tabular}{|c|c|c|}
\hline $\mathbf{T}\left({ }^{\circ} \mathbf{C}\right)$ & $\mathbf{K}\left(\mathbf{m i n}^{-1}\right)$ & $\mathbf{N}$ \\
\hline 33 & 0,0062 & 1,256 \\
\hline 41 & 0,0121 & 1,214 \\
\hline 50 & 0,0161 & 1,216 \\
\hline 66 & 0,0322 & 1,174 \\
\hline 80 & 0,0487 & 1,140 \\
\hline 92 & 0,0506 & 1,243 \\
\hline
\end{tabular}


A Figura 8 apresenta o gráfico de resíduos para as temperaturas de 33 a $92 \pm 1^{\circ} \mathrm{C}$ para o modelo de Page (1949).

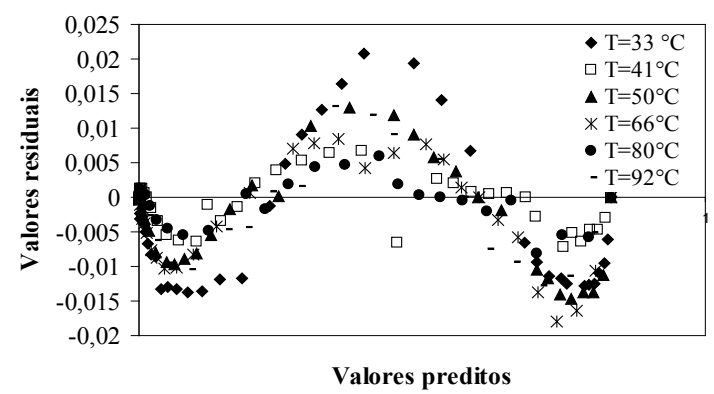

Figura 8. Resíduos estimados nas temperaturas de 33 a $92 \pm 1{ }^{\circ} \mathrm{C}$, para o modelo de Page (1949)

Como se observa, os dados residuais tiveram desvios máximos de 0,02 e mínimos de - 0,015, mas, com uma distribuição não uniforme dos resíduos, mostrando uma tendência em formato " $S$ " do comportamento, que é função da própria estrutura/ forma da equação, MOTTA LIMA et al. (2002).

\subsection{CURVAS GENERALIZADAS}

Como observado na Figura 8, o gráfico de resíduos do modelo de Page (1949) não obteve uma boa distribuição. Desta forma, optou-se, por um novo ajuste testando-se um expoente variável para o tempo (t) na equação de Page (1949), procurando assim, corrigir o formato em " $\mathrm{S}$ " da curva ajustada, conforme proposto por MOTTA LIMA et al. (2002). A Equação 15 mostra a correção linear do expoente do modelo de Page (1949) e denominado de Page Modificado.

$$
X_{R}=\exp \left(-k t^{n_{1} t+n_{2}}\right)
$$

em que $\mathrm{n}_{1}$ e $\mathrm{n}_{2}$ são parâmetros da equação a serem determinados, adimensional.

A Tabela 3 mostra os valores dos parâmetros obtidos com o ajuste da Equação 15, bem como dos dados estatísticos, para o modelo de Page Modificado.

Tabela 3. Valores estimados dos parâmetros para o modelo de Page modificado

\begin{tabular}{|c|c|c|c|c|c|c|c|c|}
\hline $\mathrm{T}\left({ }^{\circ} \mathrm{C}\right)$ & $\begin{array}{c}\mathbf{X}_{\mathbf{0}} \\
(\mathbf{g ~ \mathbf { ~ H }} \mathbf{\mathbf { O }} / \mathbf{g} \\
\text { sól. seco) }\end{array}$ & $\begin{array}{c}\mathbf{X}_{\mathbf{f}} \\
\left(\mathbf{g} \mathbf{H}_{\mathbf{2}} \mathbf{O} / \mathbf{g}\right. \\
\text { sól. seco) }\end{array}$ & $\mathbf{k}\left(\mathbf{m i n}^{-1}\right)$ & $\mathbf{n}_{\mathbf{1}}$ & $\mathbf{n}_{\mathbf{2}}$ & Teste $\mathbf{F}$ & $\mathbf{R M S E}$ & $\mathbf{R}^{\mathbf{2}}$ \\
\hline 33 & 1,00 & 0,00 & 0,0108 & 0,00078 & 1,0636 & 4147,8 & 0,0032 & 0,9994 \\
\hline 41 & 1,00 & 0,00 & 0,0135 & 0,00028 & 1,1698 & 38151,8 & 0,0028 & 0,9999 \\
\hline 50 & 1,00 & 0,00 & 0,0218 & 0,00115 & 1,0839 & 23383,8 & 0,0035 & 0,9999 \\
\hline 66 & 1,00 & 0,00 & 0,0410 & 0,01753 & 1,0607 & 26557,9 & 0,0032 & 0,9999 \\
\hline 80 & 1,00 & 0,00 & 0,0480 & 0,00263 & 1,1367 & 17021,7 & 0,0038 & 0,9998 \\
\hline 92 & 1,00 & 0,00 & 0,0518 & 0,00074 & 1,1040 & 44074,5 & 0,0023 & 0,9999 \\
\hline
\end{tabular}

Observa-se na Tabela 3 que à medida que se aumenta a temperatura, há um aumento de $\mathrm{k}$, para o modelo de Page modificado, sendo que os valores do teor de umidade de equilíbrio iniciais e finais foram 1 e 0 , respectivamente. Em relação aos testes estatísticos, nota-se que os valores do Teste F, RMSE e $\mathrm{R}^{2}$ foram melhores do que aqueles obtidos para o modelo de Page, apresentados na Tabela 1, demonstrando que a correção linear realizada no expoente do modelo de Page modificado apresentou um melhor ajuste.

A Figura 9 mostra o gráfico de resíduos para Page Modificado.

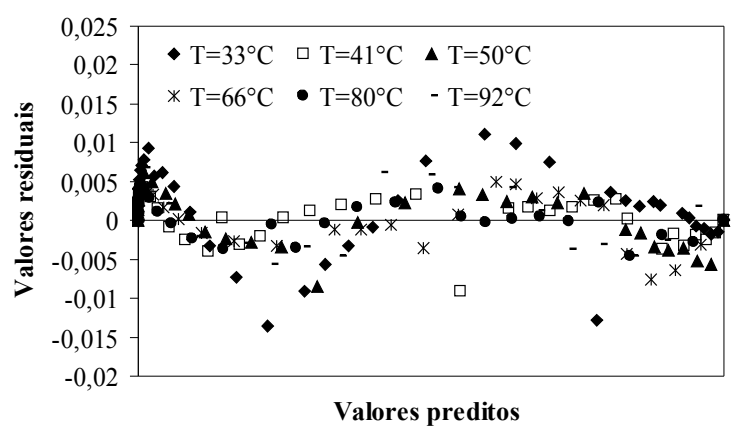

Figura 9. Resíduos estimados nas temperaturas de 33 a $92 \pm 1^{\circ} \mathrm{C}$, para o modelo de Page Modificado 
Os resíduos obtidos para o modelo de Page Modificado, mesmo apresentando a mesma tendência do modelo de Page (1949), com a utilização de novos parâmetros na equação, conseguiu suavizar a tendência do modelo, e com isto seus erros sistemáticos. Os valores residuais variaram entre valores máximos e mínimos de 0,01 e-0,01, respectivamente. Desta forma, pode-se utilizar este modelo em outras aplicações que envolvam estes intervalos.

\subsection{DETERMINAÇÃO DA DIFUSIVIDADE EFETIVA E DA ENERGIA DE ATIVAÇÃO}

O coeficiente de difusividade efetiva $\left(\mathrm{D}_{\text {eff }}\right)$ foi calculado pelas Equações 13 e 14, assumindo que a metade da espessura (L) da amostra durante o período de taxa decrescente é $0,005 \mathrm{~m}$. $\mathrm{A} \mathrm{D}_{\text {eff }}$ foi estimada por regressão não linear (método Quasi-Newton) com o auxílio do software Statistica 6.0, com um critério de convergência de 0,0001. A Figura 10 apresenta os valores da difusividade para cada temperatura testada.

À medida que ocorre um aumento na temperatura de secagem nos dados apresentados na Figura 10, verifica-se um aumento da difusividade, uma vez que a difusividade representa a velocidade com que a água migra do interior para a superfície do material, sendo então vaporizada.

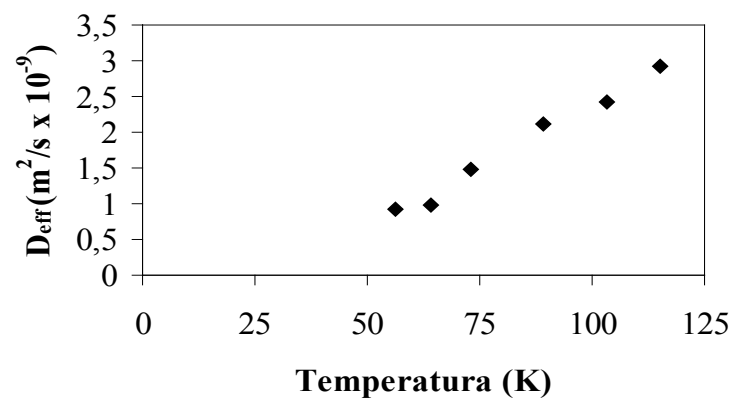

Figura 10. Efeito da temperatura na difusividade efetiva

Os valores da difusividade para as temperaturas de $33,41,50,66,80$ e $92 \pm 1^{\circ} \mathrm{C}$ foram de $9,247.10^{-10}, 9,730.10^{-10}, 1,48.10^{-9}, 2,113.10^{-9}$, $2,432.10^{-9}, 2,918.10^{-9} \mathrm{~m}^{2} \mathrm{~s}^{-1}$, respectivamente. Segundo MADAMBA (2003) os valores do coeficiente de difusividade para materiais alimentícios encontram-se na faixa de $10^{-11}$ a $10^{-9} \mathrm{~m}^{2} \mathrm{~s}^{-1}$.

Linearizando a Equação 14, determinou-se, por regressão não linear (método Quasi-Newton) com o auxílio do software Statistica $6.0^{\circledR}$, um valor para $\mathrm{D}_{0}$ igual a $9,647.10^{-8} \mathrm{~m}^{2} \mathrm{~s}^{-1}$ e para $\mathrm{E}_{\text {act }}$ de $10,669 \mathrm{KJ} \mathrm{mol}^{-1}$.

\section{CONCLUSÕES}

Com base nos resultados obtidos, observou-se que o processo de secagem do bagaço da laranja apresentou um período de taxa constante de secagem, seguido por um período de taxa decrescente, comportamento este, característico do material. Dos modelos testados, o modelo matemático de Page Modificado foi o que melhor se ajustou aos dados experimentais, pois apresentou os melhores resultados para os critérios estatísticos utilizados. Os valores do coeficiente de difusão efetiva aumentaram de acordo com a elevação da temperatura do ar de secagem. A dependência da difusividade em relação à temperatura foi descrita pela equação de Arrhenius, por meio da qual se obteve o valor da energia de ativação de $10,669 \mathrm{~kJ} \mathrm{~mol}^{-1}$.

\section{BIBLIOGRAFIA}

ALMEIDA, F.A.C., FONSECA, K.S., GOUVEIA, J.P.G. Secagem natural de gergelim e determinação da umidade de equilíbrio. Revista Brasileira de Engenharia Agrícola e Ambiental, v.3, n.3, p.343-348, 1999.

ARAMI, M., LIMAEE, N.Y., MAHMOODI, N.M., TABRIZI, N.S. Removal of dyes from colored textile wastewater by orange peel adsorbent: Equilibrium and Kinetic studies. Journal Colloid and Interface Science, v.288, p.371-376, 2005.

BROOKER, D.B., BAKER-ARKEMA, F.W., HALL, C.W. Drying and stoprage of grains and oilseeds. New York: AVI Book, 1992, 450p.

CORREAA, P.C., RIBEIRO, D.M., RESENDE, O., BOTELHO, F.M. Determinação e modelagem das propriedades físicas e da contração volumétrica do trigo, durante a secagem. Revista Brasileira de Engenharia Agricola e Ambiental, v.10, n.3, p.665-670, 2006.

CRANK, J., 1975. The Mathematics of Diffusion, second Ed. Oxford University Press, London. 
DANDAMRONGRAK, R., YOUNG, G., MASON, R. Evaluation of various pre-treatments for the dehydration of banana and selection of suitable drying models. Journal of Food Engineering, v.55, p.139-146, 2002.

DOYMAZ, I. Drying kinetics of black grapes treated with different solutions. Journal of Food Engineering, v. 76, p.212-217, 2006.

DOYMAZ, I. Air-drying characteristics of tomatoes. Journal of Food Engineering, v.78, p.12911297, 2007.

ERTEKIN, C., YALDIZ, O. Drying of eggplant and selection of a suitable thin layer drying model. Journal of Food Engineering, v.63, p.349-359, 2004.

FIORENTIN, L.D., TRIGUEROS D.E.G., MÓDENES, A.N. QUINONES F.R.E., PEREIRA, N.C., BARROS S.T. D., SANTOS O.A.A. Biosorption of reactive blue $5 \mathrm{G}$ dye onto drying orange bagasse in batch system: kinetic and equilibrium modeling. Chemical Engineering Journal, 163, p.68-77, 2010.

GHAZANFARI, A., EMAMI, S., TABIL, L.G., PANIGRAHI, S. Thing-layer druing of flax fiber:II. Modeling during process using semi-theoretical and empirical models. Drying Technology, v.24, p.1637-1642, 2006.

HENDERSON, S.M. Progress in developing the thin layer drying equation. Transactions of the American Society of Agricultural Engineers, St. Joseph, v. 17, n. 2, p.1167-1168, 1974.

ÍTAVO, L.C.V., SANTOS, G.T., JOBIM, C.C., VOLTOLINI, T.V., BORTOLASSI, J.R., FERREIRA, C.C. B. Aditivos na conservação do bagaço de laranja in natura na forma de silagem. Revista Brasileira de Zootecnia, v.29, n.5, p.14741484, 2000.

KINGSLY, A.R.P., SINGH, D.B. Drying kinetics of pomegranate arils. Journal of Food Engineering, v.79, p.741-744, 2007.

LEWIS, W.K. The rate of drying of solid materials. Journal of Industrial and Engineering Chemistry, v.13, n.5, p.427-432, 1921.
LUZ, G.R., ANDRADE, C.M.G., JORGE, L.M.M., PARAÍSO, P.R. Coeficiente de transferência de massa na secagem do farelo de soja. In: Congresso Brasileiro de Sistemas Particulados, ENEMP, Maringá - PR, 2006.

MADAMBA, P.S. Thin layer drying models for osmotically pré-dried Young coconut. Drying Technology, v.21, n.9, p.1759-1780, 2003.

MARTINAZZO, A.P., CORRÊA, P.C., RESENDE, O., MELO, E.C. Análise e descrição matemática da cinética de secagem de folhas de capim-limão. Revista Brasileira de Engenharia Agricola e Ambiental, v.11, n.3, p.301-306, 2007.

MWITHIGA, G., OLWAL, J.O. The drying kinetics of kale (brassica oleracea) in a convective hot air dryer. Journal of Food Engineering, v.71, p.373378, 2005.

MOTTA LIMA, O.C., PEREIRA, N.C., MENDES, E.S. Curvas generalizadas na análise da cinética de secagem de papel artesanal com ar ambiente em convecção forçada, $30^{\circ}$ ENEMP, UFSCAR, 2002.

OLIVEIRA, R.A., OLIVEIRA, W.P. De, PARK, K. J. Determinação da difusividade efetiva de raiz de chicória. Engenharia Agrícola Jaboticabal, v.26, n.1, p.181-189, 2006

ORIKASA, T., WUB, L., SHIINA, T., TAGAWA, A. Drying characteristics of kiwifruit during hot air drying. Journal of Food Engineering, v.85, p.303-308, 2008.

PAGE, G.E. Factors influencing the maximum rates of air drying shelled corn in thin layers, Indiana, 1949, Thesis (M. Sc.), Purdue University.

RIVERA, L.L., LOPEZ, R., GARCIA-ALVARADO, M.A., SALGADO-CERVANTES, M.A. Mathematical simulation of the effective diffusivity of water during drying of papaya. Drying Technology, v.25, p.1633-1638, 2007.

SIVARAJ, R., NAMASIVAYAM, C., KADIRVE$\mathrm{LU}, \mathrm{K}$. Orange peel as an adsorbent in the renoval of Acid violet 17 (acid dye) from aqueous solutions. Waste Management, v.21, p.102-110, 2001. 
SOUSA, L.H.C.D. Analysis of drying kinetics and moisture distribution in convective textile fabric drying. Drying Technology, v.24, p.485-497, 2006.

SOUSA, L.H.C.D. Estudo da Secagem de Materiais Têxteis, Maringá, 2003, Tese (Doutorado), Universidade Estadual de Maringá.

TRIPODO, M.M., LANUZZA, F., MICALI, G., COPPOLINO, R., NUCITA, F. Citrus waste recovery: a new environmentally friendly procedure to obtain animal feed. Bioresource Technology, v.91, p.111-115, 2004.
WANG, C.Y., SINGH, R.P. Use of variable equilibrium moisture content in modeling rice drying. ASAE Paper No. 78-6505, ASAE, St. Joseph, MI, 1978.

YILDIRIM, A., ONER, M. D., BAYRAM, M. Fitting Fick's model to analyze water diffusion into chickpeas during soaking with ultrasound treatment. Journal of Food Engineering, v. 104, p.134-142, 2011. 